\title{
Cerebrorenal Interaction and Stroke Outcome
}

\author{
Masatoshi Koga
}

Department of Cerebrovascular Medicine, National Cerebral and Cardiovascular Center, Suita, Osaka, Japan

\section{See article vol. 25: 570-579}

Chronic kidney disease (CKD) is a global health burden. CKD originally indicates a high-risk state of future hemodialysis and is now known as an independent contributor to cardiovascular disease and stroke. According to a recent systematic review and metaanalysis, stroke risk increases linearly and additively with declining glomerular filtration rate (GFR) and increasing albuminuria ${ }^{1)}$. In the last decade, several studies with medium sample sizes reported a significant association between CKD and stroke outcomes $^{2-4)}$. However, there have been little data showing the association within a large-scale populationbased setting.

In the present study, Nugroho and colleagues assessed the association between admission estimated GFR (eGFR) and acute stroke outcomes, including patients with and without renal replacement therapy, using the Shiga Stroke Registry ${ }^{5)}$. The Shiga Stroke Registry is a multicenter, population-based, stroke registry covering all stroke cases in Shiga Prefecture (population: 1.4 million). They showed that low eGFR $(<$ 45 vs. $60-89 \mathrm{~mL} / \mathrm{min} / 1.73 \mathrm{~m}^{2}$ ) was independently associated with in-hospital death and at-discharge death/disability (modified Rankin Scale 2-6) in ischemic stroke using a large-scale population-based database. Patients with renal replacement therapy had about a five-fold increased risk of in-hospital death compared with those with eGFR $60-89 \mathrm{~mL} / \mathrm{min} / 1.73$ $\mathrm{m}^{2}$ in hemorrhagic stroke. Interestingly, they found that high eGFR (>90 vs. $60-89 \mathrm{~mL} / \mathrm{min} / 1.73 \mathrm{~m}^{2}$ ) was positively associated with at-discharge death/disability in ischemic stroke. They also conducted a sen-

Address for correspondence: Masatoshi Koga, 5-7-1 Fujishirodai, Suita, Osaka 565-8565, Japan

E-mail: koga@ncvc.go.jp

Received: December 29, 2017

Accepted for publication: January 9, 2018 sitivity analysis using a modified Rankin Scale 3-6 as the unfavorable outcome and found similar trends. The strong point of this study was that a large-scale population-based database was used and they included patients with and without renal replacement therapy. The weak points in this study were as follows. The Japan Coma Scale (JCS) is not familiar to people, except for Japanese physicians and researchers, although the JCS is a well-validated scale for predicting stroke outcome. In addition, the timing of the outcome (modified Rankin Scale) measure was not uniform because an at-discharge outcome was used.

As the authors have mentioned, the mechanism underlying the relationship between renal function and poor stroke outcome has not been clearly understood. Because CKD and stroke share cardiovascular risk factors and these patients are likely to have multiple risk factors, an overlap of traditional risk factors (aging, hypertension, diabetes, dyslipidemia, obesity, and smoking) and nontraditional risk factors (chronic inflammation, asymmetric dimethylarginine, oxidative stress, sympathetic nerve overactivity, thrombogenic factors, and hyperhomocysteinemia) could trigger large infarcts with severe clinical symptoms and a tendency to stroke progression ${ }^{6}$. Albuminuria is associated with increased inflammatory cytokines and oxidative stress, which could cause excessive vascular damage, hemorrhagic transformation, and cerebral edema. Furthermore, fatal and unfavorable functional outcomes in patients with CKD might be partly attributed to acute kidney injury because CKD is a predictor of acute kidney injury. Although there are some studies showing a significant association between high eGFR and cardiovascular events in non-stroke patients ${ }^{7,8)}$, the association between high eGFR and unfavorable outcome is still inconclusive, and we need more data in acute stroke.

Now, we recognize that CKD is an independent predictor of stroke outcome. Although the authors have discussed that a more vigilant management of acute 
stroke patients with CKD is warranted, there is no evidence to show what the effective management is so far. I hope that safe and effective managements for stroke patients with CKD will be developed and established in the near future.

\section{Conflicts of Interest}

None.

\section{References}

1) Masson P, Webster AC, Hong M, Turner R, Lindley RI, Craig JC: Chronic kidney disease and the risk of stroke: a systematic review and meta-analysis. Nephrol Dial Transplant, 2015; 30: 1162-1169

2) Naganuma M, Koga M, Shiokawa Y, Nakagawara J, Furui E, Kimura K, Yamagami H, Okada Y, Hasegawa Y, Kario K, Okuda S, Nishiyama K, Minematsu K, Toyoda K: Reduced estimated glomerular filtration rate is associated with stroke outcome after intravenous rt-PA: the Stroke Acute Management with Urgent Risk-Factor Assessment and Improvement (SAMURAI) rt-PA registry. Cerebrovasc Dis, 2011; 31: 123-129

3) Miyagi T, Koga M, Yamagami H, Okuda S, Okada Y, Kimura K, Shiokawa Y, Nakagawara J, Furui E, Hasegawa
Y, Kario K, Arihiro S, Sato S, Minematsu K, Toyoda K: Reduced estimated glomerular filtration rate affects outcomes 3 months after intracerebral hemorrhage: the stroke acute management with urgent risk-factor assessment and improvement-intracerebral hemorrhage study. J Stroke Cerebrovasc Dis, 2015; 24: 176-182

4) Kumai Y, Kamouchi M, Hata J, Ago T, Kitayama J, Nakane H, Sugimori H, Kitazono T, Investigators FSR: Proteinuria and clinical outcomes after ischemic stroke. Neurology, 2012; 78: 1909-1915

5) Nugroho AW, Arima H, Miyazaki I, Fujii T, Miyamatsu N, Sugimoto Y, Nagata S, Komori M, Takashima N, Kita Y, Miura K, Nozaki K: The association between glomerular filtration rate estimated on admission and acute stroke outcome: the Shiga Stroke Registry. J Atheroscler Thromb, 2018; 25: 570-579

6) Toyoda K, Ninomiya T: Stroke and cerebrovascular diseases in patients with chronic kidney disease. Lancet neurology, 2014; 13: 823-833

7) Park M, Yoon E, Lim YH, Kim H, Choi J, Yoon HJ: Renal hyperfiltration as a novel marker of all-cause mortality. J Am Soc Nephrol, 2015; 26: 1426-1433

8) Reboldi G, Verdecchia P, Fiorucci G, Beilin LJ, Eguchi K, Imai Y, Kario K, Ohkubo T, Pierdomenico SD, Schwartz JE, Wing L, Saladini F, Palatini P: Glomerular hyperfiltration is a predictor of adverse cardiovascular outcomes. Kidney Int, 2018; 93: 195-203 